\title{
The effects of contig length and depth on the estimation of SNP frequencies, and the relative abundance of SNPs in protein-coding and non-coding transcripts of tiger salamanders (Ambystoma tigrinum)
}

\author{
Soo Hyung Eo ${ }^{1,3^{*}}$ and J Andrew DeWoody $y^{1,2}$
}

\begin{abstract}
Background: Next-generation sequencing methods have contributed to rapid progress in the fields of genomics and population genetics. Using this high-throughput and cost-effective technology, a number of studies have estimated single nucleotide polymorphism (SNP) frequency by calculating the mean number of SNPs per unit sequence length (e.g., mean SNPs/kb). However, both read length and contig depth are highly variable and thus raise doubt about simple methods of SNP frequency estimation.

Results: We used 454 pyrosequencing to identify 2,980 putative SNPs in the eastern tiger salamander (Ambystoma tigrinum tigrinum) transcriptome, then constructed analytical models to estimate SNP frequency. The model which considered only contig length (i.e., the method employed in most published papers) was evaluated with very poor likelihood. Our most robust model considered read depth as well as contig length, and was $7.5 \times 10^{55}$ times more likely than the length-only model. Using this novel modeling approach, we estimated SNP frequency in proteincoding (mRNA) and non-coding transcripts (e.g., small RNAs). We found little difference in SNP frequency in the contigs, but we found a trend of a higher frequency of SNPs in long contigs representing non-coding transcripts relative to protein-coding transcripts. These results support the hypothesis that long non-coding transcripts are less conserved than long protein-coding transcripts.

Conclusions: A modeling approach (i.e., using multiple model construction and model selection approaches) can be a powerful tool for identifying selection on specific functional sequence groups by comparing the frequency and distribution of polymorphisms.
\end{abstract}

Keywords: Contig depth, Contig length, Model selection, SNP frequency, Transcriptome, 454 sequencing

\section{Background}

Mutations and subsequent polymorphisms are important not only for identifying influences on genetic disease $[1,2]$, but also for understanding selection, adaptation, and other evolutionary processes $[3,4]$. While some new mutants have higher fitness than others and therefore are

\footnotetext{
* Correspondence: eos@purdue.edu

'Department of Forestry \& Natural Resources, Purdue University, West Lafayette, IN 47907, USA

${ }^{3}$ Current address: Department of Zoology, University of Wisconsin, Madison, WI 53706, USA

Full list of author information is available at the end of the article
}

retained by positive selection, most mutations are quickly removed by purifying selection because they are deleterious $[5,6]$. The intensity of selection may differ across genomic locations (e.g., genic and intergenic regions) and thus nucleotide polymorphisms have different evolutionary impacts depending on their genomic locations. Single nucleotide polymorphism (SNP) profiles can be used to infer the intensity of selection by comparing the frequency and distribution of SNPs of two or more sequence regions, such as genic vs. intergenic regions, exonic vs. intronic vs. untranslated regions, autosomes 
vs. sex chromosomes, and particular gene groups with specific functions [7-9].

To properly compare SNP frequency among groups (e.g., among functional gene groups, among chromosomes, among populations), large samples of similar size are desirable. For example, the International HapMap Consortium $[10,11]$ estimated the frequency and distribution of human SNPs at the genome-wide level; they compared these among three geographically diverse populations by genotyping 90 individuals from each population. To date, such large-scale, systematic evaluations have been limited to human or a few model species e.g., [12].

The advent of high-throughput sequencing technologies (e.g., 454, SOLiD, and Illumina) has contributed to the rapid development of SNPs in non-model but important wild species $[7,9,13,14]$. In the context of nextgeneration sequencing, SNP discovery in non-model species is usually achieved by comparing the consensus sequence of a contig with the individual sequences that comprise the contig. However, it is not easy to estimate the frequency of such SNPs because of extreme variation in contig read length and depth; we are more likely to observe SNPs in a $10 \mathrm{~kb}$-long-sequence than in a $1 \mathrm{~kb}$ long-sequence. Furthermore, we have a higher probability of identifying SNPs when we compare contigs comprised of 100 reads than with those comprised of 2 sequence reads. Despite these multiple factors, most next-gen analyses estimate SNP frequency simply by determining the average number of SNPs per unit sequence length (e.g., mean $\mathrm{SNP} / \mathrm{kb}$ ), thus ignoring bias due to contig depth. One of our goals was to determine if such length-only-methods can effectively estimate SNP frequency from next-gen datasets or if more sophisticated approaches are needed.

A second goal was to compare SNP frequencies in protein-coding transcripts $v s$. non-coding transcripts. A "transcriptome" consists of transcripts not only from protein-coding mRNA but also from non-coding RNAs such as ribosomal RNA (rRNA) and transfer RNA (tRNA). In general, non-coding transcripts are expected to be less conserved (i.e., more polymorphic) than protein-coding regions because selection may eliminate deleterious mutations from functional sequences $[15,16]$. However, recent transcriptome studies have shown that there are many types of non-protein-coding RNAs with functions of biological significance such as chromatin modification and transcriptional regulation [17]. This may imply that at least some non-coding transcripts are as well conserved as protein-coding transcripts. However, (to our knowledge) no study has systematically evaluated SNP frequencies in coding and non-coding transcripts at the transcriptome level. Although there are some reports that long non-protein-coding RNAs in humans and mice have evolved rapidly [18-20], the data are still lacking-especially in non-mammalian species.

One of our ongoing research programs is focused on evolutionary and ecological genomics of tiger salamanders [21-24]. For this research, we sequenced the transcriptome of the eastern tiger salamander (A. t. tigrinum) using 454 pyrosequencing and then analyzed the distribution and frequency of SNPs. We studied the influence of contig depth on the estimation of SNP frequencies, and we tested the hypothesis that non-coding transcripts are less conserved than protein-coding transcripts by comparing their SNP frequencies.

\section{Methods}

\section{Sample preparation and 454 transcriptome sequencing}

We captured and sampled seven tiger salamanders at the Purdue Wildlife Area (Indiana, USA) consistent with IACUC 1203000614 and in accordance with guidelines by the American Society of Ichthyologists and Herpetologists and by the American Veterinary Medical Association (June 2007). RNA was extracted from gill, lung, skin and spleen using TRIzol ${ }^{\circledR}$ reagent (Invitrogen). We constructed a total of 19 tissue-specific cDNA libraries from these RNA samples using the ClonTech SMART cDNA synthesis kit, utilizing a modified poly-T primer [25]. Each double-stranded cDNA library was digested with $S f i$ to remove excess primers and subsequently purified with the QIAquick PCR purification kit (Qiagen). Approximately $5 \mu \mathrm{g}$ of each cDNA library was sequenced using a 454 Genome Sequencer with FLX Titanium chemistry (454 Life Sciences). Briefly, the cDNA fragments were sheared via nebulization, hybridized to DNA capture beads, and amplified by emulsion-based PCR. Molecular barcodes with multiplex identifiers (MIDs, unique $10 \mathrm{bp}$ sequences) were applied to specifically tag each cDNA library in our pooled sequencing run.

\section{Contig assembly and SNP detection}

We pooled all sequences from seven individuals in PCAP [26] and assembled contigs from the entire data set using default settings with the overlap cutoff of $92 \%$ identity. The consensus sequence from each of these contigs was used as a reference sequence to which individual sequence reads were aligned using PCAP. First, we identified SNPs only from contigs at least $100 \mathrm{bp}$ in length and with a depth of 10 or more reads. We limited SNP scoring to contigs which have minor allele frequency of 0.01 or at least two minor allele reads. Furthermore, we limited SNPs to regions where $20 \mathrm{bp}$ of high-quality sequence data was present both upstream and downstream of the variable site. We discounted SNPs in homopolymer repeats of $>4$ nucleotides, and defined high-quality sites as those with a PCAP base quality score $>20$. We considered only biallelic SNP 
substitutions, excluding all indels and triallelic sites. Second (in a separate analysis), we identified SNPs in long contigs (at least $501 \mathrm{bp}$ ) [27] using otherwise identical methods. These long contigs are thus a subset of all contigs. Our use of these two datasets ("all" contigs of $\geq 100 \mathrm{bp}$ and "long" contigs of $>500 \mathrm{bp}$ ) allowed us to evaluate SNP frequencies in long transcripts.

\section{Discriminating among protein-coding and non-protein- coding transcripts}

We divided all contigs into categories of protein-coding and non-coding transcripts using the program CPC [28]. $\mathrm{CPC}$ employs supervised learning algorithms known as support vector machines to discriminate between proteincoding and non-protein-coding transcripts $[28,29]$. The program assessed the protein-coding potential scores of each contig based on the quality of the predicted open reading frame (ORF) and on the quality of the BLAST hit against UniProt Reference Clusters. CPC was used to determine ORF quality by log-odds score, coverage, and integrity of the ORF whereas BLAST hit quality was determined by BLAST hit number, feature of high-scoring segment pairs (HSP), and HSP frame score among three reading frames. Positive coding potential values reflect protein-coding transcripts whereas negative coding potential values designate non-protein-coding transcripts. Thus, we distinguished protein-coding from non-coding transcripts by considering coding potential scores of a contig (and by checking the score of its reverse complement strand).

\section{Modeling SNP frequencies and comparisons between protein-coding and non-coding transcripts}

Our first goal was to evaluate the effects of contig length and depth on the estimated frequency of SNPs. A priori, we predicted that SNP frequency would positively associate with both factors. We employed the informationtheoretic approach suggested by Akaike [30] and extended by Burnham and Anderson [31] to evaluate the relative plausibility of models relating contig length and depth to the number of SNPs in contigs. Our second goal was to test the hypothesis that SNP frequency in non-coding transcripts is higher than in protein-coding transcripts. To test this, we included transcript type (protein-coding or non-coding) as a variable in all models. Therefore, the global model consisted of length (LENGTH, the number of bp), depth (DEPTH, the number of aligned sequence reads), and the variable of transcript type $(\mathrm{C} / \mathrm{NC}$, coded with 1 for protein-coding transcript and 0 for non-coding transcript) of each contig. We used SAS 9.2 to estimate parameter values.

Four candidate models were fitted using regression models based on a negative binomial distribution. To assess the relative fit of each model, we compared the
Akaike's Information Criteria (AIC), with better fitting models having lower AIC [31]. The relative plausibility or weight of each model was evaluated by measuring Akaike weight $\left(w_{i}\right)$. We repeated construction of candidate models and model selection for the number of transitions $\left(T_{i}\right)$ and transversions $\left(T_{v}\right)$ as the response variables, as well as for the number of SNPs in a contig. For ease of interpretation of the relative effect of each variable, we plotted estimates of the relationships between length or depth of contigs and the number of SNPs found in protein-coding and non-coding transcripts.

\section{Results}

Our 454 pyrosequencing run on the cDNA from seven tiger salamanders yielded a total of 670,408 sequence reads spanning $190.4 \mathrm{Mb}$. After quality control and other filtering (e.g., repetitive elements, short reads, etc.) we assembled the remaining 273,501 high-quality sequences into 51,391 contigs. Among contigs with at least 10 sequence reads, 4,552 and 3,181 contigs were $\geq 100$ and $\geq 501 \mathrm{bp}$ in length, respectively (Table 1 ; Additional file 1). In the "all" contig dataset of $\geq 100$-bp contigs, protein-coding transcripts were slightly more rare than non-coding transcripts $(2,146 v s .2,406)$; the pattern was reversed in the "long" dataset of $\geq 501$-bp contigs where protein-coding transcripts were more common (1,762 vs. 1,419). A total of 2,980 SNPs that passed our stringent scoring criteria were detected in the "all" contig dataset; of these, 2,515 (84\%) were found in the "long" dataset (Additional file 1). The ratio $T_{i} / T_{\nu}$ was 1.8 in both datasets. An overview of the assembled contigs and SNPs is presented in Table 1 and our short read

Table 1 Summary statistics of assembled contigs (with depth of $\geq 10$ reads) and SNPs

\begin{tabular}{ccll}
\hline & Total & $\begin{array}{l}\text { Protein-coding } \\
\text { transcript }\end{array}$ & $\begin{array}{l}\text { Non-coding } \\
\text { transcript }\end{array}$ \\
\hline Contigs (with length of 100 bp or longer) & & \\
Number of cocntigs & 4552 & 2146 & 2406 \\
Mean length (bp) & 811 & 977 & 664 \\
Mean depth & 28 & 36 & 21 \\
Number of SNPs & 2980 & 1744 & 1236 \\
$\quad$ Number of transitions & 1917 & 1139 & 778 \\
Number of transversions & 1063 & 605 & 458 \\
Contigs (with length of 501 bp or longer) & & \\
Number of contigs & 3181 & 1762 & 1419 \\
Mean length (bp) & 1003 & 1095 & 889 \\
Mean depth & 32 & 39 & 23 \\
Number of SNPs & 2515 & 1555 & 960 \\
Number of transitions & 1603 & 1009 & 594 \\
Number of transversions & 912 & 546 & 366 \\
\hline
\end{tabular}


sequence data will be deposited at Dryad (http://dx.doi. org/10.5061/dryad.5pd17).

The correlation results were qualitatively similar between the "all" (Additional file 2: Figure S1) and "long" datasets (Figure 1). Here we focus on the long dataset, where the number of SNPs observed in a contig averaged 0.79 and ranged from 0 to 26. Mean contig length in the long dataset was 1,003 bp (range 501 $5,354 \mathrm{bp}$ ) and mean contig depth was 32 reads (range 10-315). We found positive associations between the number of SNPs and contig length $(r=0.32 ; P<0.001$; Figure 1A), and between the number of SNPs and contig depth $(r=0.46 ; P<0.001 ;$ Figure 1B). These same trends were apparent in both protein-coding $(r=0.36$ for length $v$ s. SNP frequency; $r=0.47$ for depth vs. SNP frequency) and non-coding transcripts $(r=0.22$ for length $v$ s. SNP frequency; $r=0.44$ for depth vs. SNP frequency); all of these associations were significant $(P<0.001)$.

To estimate SNP frequency in the 'long' ( $\geq 501 \mathrm{bp}$ and $\geq 10$ reads) contig dataset, we used the negative binomial
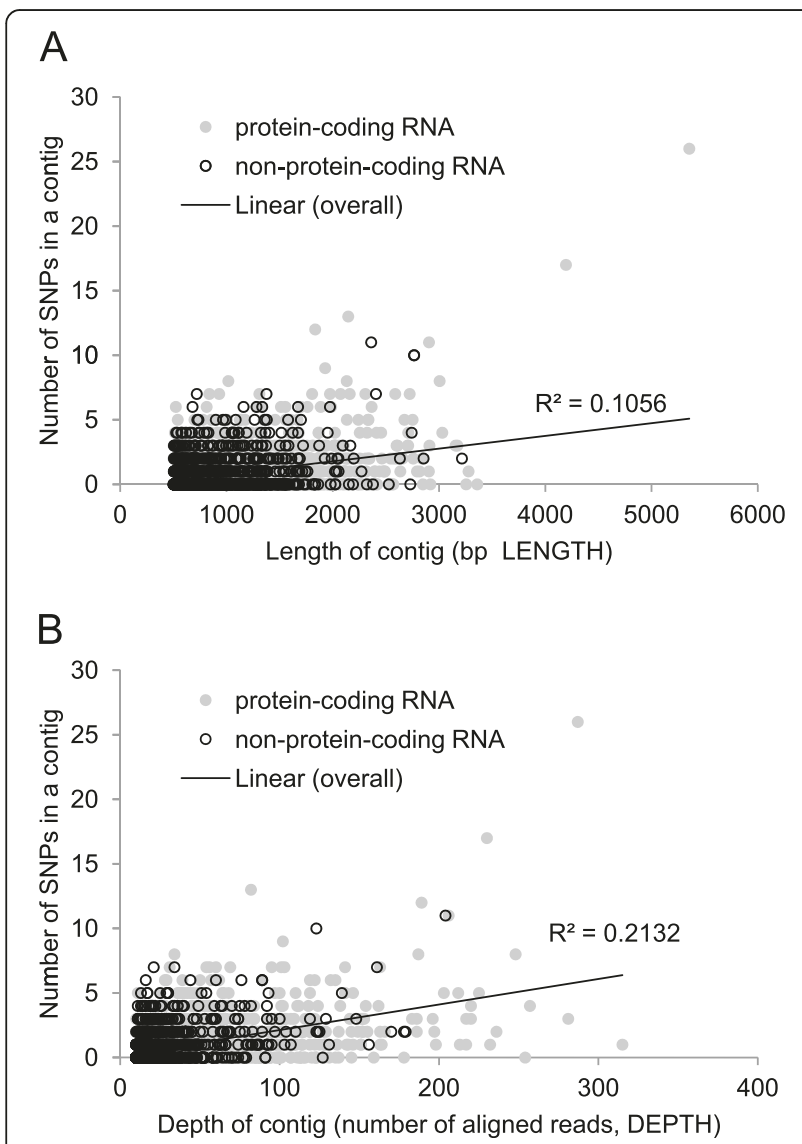

Figure 1 Correlations between the number of SNPs and both length and depth of contigs. Positive correlations between the number of SNPs and both (A) length and (B) depth of contigs with depth of $\geq 10$ reads and length of $\geq 501$ bp. In both cases, $P<0.001$. distribution to construct four regression models that considered contig length, contig depth, and the category of transcript (protein-coding and non-coding transcript). The most robust model of SNP frequency was the global model that used all of these variables; similarly, the global models were most robust for estimating the frequency of transitions $\left(T_{i}\right)$ and transversions $\left(T_{v}\right)$ (Table 2). The $w_{i}$ (relative plausability or weight of each model) of the global models for estimating the frequency of SNPs, $T_{i}$, and $T_{v}$, ranged from 0.68 to 0.99. In sharp contrast, those of the commonly-employed "length-butno-depth" models ranged from $6.7 \times 10^{-24}$ to $1.2 \times 10^{-56}$. Our best-fit model predicting SNP frequency was $7.5 \times 10^{55}\left(=0.904 /\left(1.2 \times 10^{-56}\right)\right)$ times more likely than the length-but-no-depth model (Table 2). Note the depth-but-no-length was also a much better model than the commonly employed length-but-no-depth model (Table 2). The "all" contig dataset yielded qualitatively similar results (Additional file 2: Table S1).

Estimation based on the best-fit model (Table 3) indicated that SNP frequency increased with contig length

Table 2 Model selection among candidate models predicting frequencies of SNPs, transition and transversion in contigs

\begin{tabular}{|c|c|c|c|c|}
\hline Model & Parameters $^{\mathrm{a}}$ & AIC & $\Delta \mathrm{AIC}^{\mathrm{b}}$ & $w_{i}^{c}$ \\
\hline \multicolumn{5}{|c|}{ for estimating the number of SNPS } \\
\hline $\begin{array}{l}\text { M1 (best, } \\
\text { full model) }\end{array}$ & $\begin{array}{l}\text { Intercept**, } \\
\text { LENGTH*, DEPTH**, C/NC+ }\end{array}$ & 7283.4 & 0.0 & 0.904 \\
\hline M2 & Intercept**, DEPTH ${ }^{* *}, \mathrm{C} / \mathrm{NC}$ & 7287.9 & 4.5 & 0.096 \\
\hline M3 & Intercept ${ }^{* *}$, LENGTH$^{* *}, \mathrm{C} / \mathrm{NC}$ & 7540.7 & 257.3 & $1.2 \times 10^{-56}$ \\
\hline M4 & Intercept ${ }^{* *}, \mathrm{C} / \mathrm{NC}^{* *}$ & 7748.3 & 464.9 & $1.0 \times 10^{-101}$ \\
\hline
\end{tabular}

for estimating the number of transitions $\left(T_{i}\right)$

$\begin{array}{llrll}\text { M1 (best, } & \text { Intercept**, } & 5682.4 & 0.0 & 0.681 \\ \text { full model) } & \text { LENGTHt, DEPTH }{ }^{* *}, \text { C/NC } & & & \\ \text { M2 } & \text { Intercept**, DEPTH**, C/NC } & 5683.9 & 1.5 & 0.319 \\ \text { M3 } & \text { Intercept**, LENGTH }{ }^{* *}, \text { C/NC+ } & 5896.1 & 213.7 & 2.7 \times 10^{-47} \\ \text { M4 } & \text { Intercept**, C/NC }{ }^{* *} & 6061.0 & 378.5 & 6.3 \times 10^{-83}\end{array}$

for estimating the number of transversions $\left(T_{v}\right)$

\begin{tabular}{|c|c|c|c|c|}
\hline $\begin{array}{l}\text { M1 (best, } \\
\text { full model) }\end{array}$ & $\begin{array}{l}\text { Intercept**, LENGTH**, } \\
\text { DEPTH }{ }^{* *}, C / \text { NC }^{*}\end{array}$ & 4077.1 & 0.0 & 0.991 \\
\hline M2 & Intercept ${ }^{* *}, \mathrm{DEPTH} \mathrm{H}^{* *}, \mathrm{C} / \mathrm{NC}+$ & 4086.6 & 9.5 & 0.009 \\
\hline M3 & Intercept ${ }^{* *}$, LENGTH $^{* *}, \mathrm{C} / \mathrm{NC}$ & 4183.8 & 106.7 & $6.7 \times 10^{-24}$ \\
\hline M4 & Intercept**, C/NC* & 4310.4 & 233.3 & $2.2 \times 10^{-51}$ \\
\hline
\end{tabular}

Model selection among candidate regression models using negative binomial distribution predicting the frequency of SNPs, transitions, and transversions in contigs (depth of 10 reads or more; length of 501 bp or longer), using Akaike's Information Criteria (AIC).

${ }^{a}$ LENGTH and DEPTH are the length and the depth of contigs, respectively, and $\mathrm{C} / \mathrm{NC}$ is a dummy variable for the type of transcript (protein-coding (coded with 1) vs non-coding transcript (coded with 0 ). ${ }^{* *}, P<0.01 ;{ }^{*}, P<0.05$; $t, P<0.1)$ variable in each model.

${ }^{\mathrm{b}} \triangle \mathrm{AIC}$ is the difference between the AIC of the best fitting model and that of each model.

${ }^{c} w_{i}$ is Akaike weight of each model. 
Table 3 Parameter estimates from best fitting model predicting frequencies of SNPs, transition and transversions in contigs

\begin{tabular}{ccrrr}
\hline Parameter $^{\mathbf{a}}$ & Estimate & \multicolumn{2}{c}{ 95\% confidence limits } & \multicolumn{1}{l}{ P } \\
\hline for estimating the number of SNPS & & & \\
Intercept & -0.9828 & -1.1137 & -0.8520 & $<0.000$ \\
LENGTH & 0.0002 & 0.0000 & 0.0003 & 0.0106 \\
DEPTH & 0.0146 & 0.0127 & 0.0165 & $<0.000$ \\
C/NC & -0.0999 & -0.2136 & 0.0139 & 0.0854
\end{tabular}

for estimating the number of transitions $\left(T_{i}\right)$

$\begin{array}{lrrrr}\text { Intercept } & -1.4039 & -1.5489 & -1.2588 & <0.0001 \\ \text { LENGTH } & 0.0001 & 0.0000 & 0.0003 & 0.0594 \\ \text { DEPTH } & 0.0139 & 0.0119 & 0.0158 & <0.0001 \\ \text { C/NC } & -0.0398 & -0.1683 & 0.0886 & 0.5435\end{array}$

for estimating the number of transversions $\left(T_{v}\right)$

\begin{tabular}{lrrrr} 
Intercept & -2.0007 & -2.1868 & -1.8147 & $<0.0001$ \\
LENGTH & 0.0003 & 0.0001 & 0.0005 & 0.0006 \\
DEPTH & 0.0123 & 0.0099 & 0.0147 & $<0.0001$ \\
C/NC & -0.1883 & -0.3539 & -0.0226 & 0.0259 \\
\hline
\end{tabular}

Estimates of variables from best fitting candidate model predicting the frequency of SNPs, transitions, and transversions in contigs (depth of 10 reads or more; length of $501 \mathrm{bp}$ or longer).

a LENGTH and DEPTH are the length and the depth of contigs, respectively, and $\mathrm{C} / \mathrm{NC}$ is a dummy variable for the type of transcript (protein-coding (coded as 1 ) vs non-coding transcript (coded as 0 )).

and depth in both protein-coding and non-coding transcripts. Using the long dataset, we estimated a marginally higher frequency of SNPs in non-coding transcripts than in protein-coding transcripts $(\mathrm{C} / \mathrm{NC}$ coefficient of $-0.09 ; P=0.08)$ but this trend was not apparent in the "all" contig dataset $(\mathrm{C} / \mathrm{NC}$ coefficient of $0.04 ; P=0.43$; Additional file 2: Table S2; Additional file 2: Figure S2). From this model, we estimated the number of SNPs in the long dataset as 0.48 (protein-coding) and 0.52 (noncoding) in $1 \mathrm{~kb}$-long contigs with a coverage depth of 10 reads, respectively (Figure 2). Similarly, we predicted 1.78 (protein-coding) and 1.97 (non-coding) SNPs in 1 -kb long contigs with a depth of 100 reads, respectively.

\section{Discussion}

We used 454 pyrosequencing to generate $190 \mathrm{Mbp}$ of tiger salamander transcriptome sequences that contained 2,980 putative SNPs. These high quality candidate SNPs, identified using strict criteria, can be converted into genetic markers for studies of natural selection, genomic organization, and allele-specific expression [14,32-34]. We used these data to develop new models for the estimation of SNP, $T_{i}$, and $T_{v}$ frequency; we then compared these estimates in coding and non-coding transcripts as discussed below.

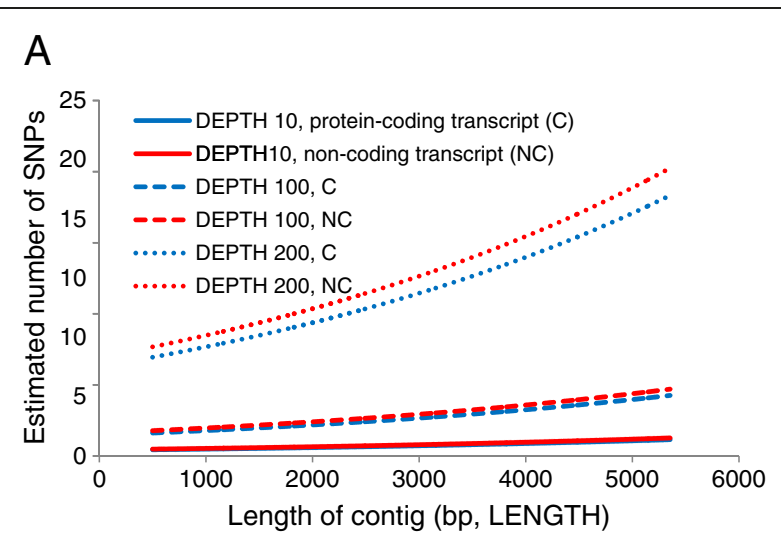

B

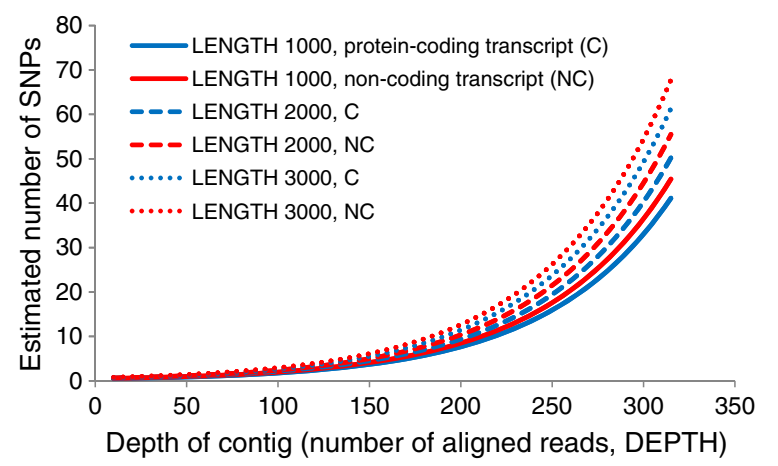

Figure 2 Estimates of the number of SNPs in transcripts based on length and depth of contigs. Estimates and comparison of the number of SNPs in protein-coding and non-coding transcripts based on various length and depth of contigs (depth of $\geq 10$ reads; length of $\geq 501$ bp), using best fitting models (Table 3 ).

Modeling frequency estimates of SNP, $T_{i}$, and $T_{v}$

A number of recent studies have estimated SNP frequency using high-throughput sequencing technology by calculating the mean number of SNPs per unit sequence length. For example, Vera et al. [14] estimated a frequency of $6.7 \mathrm{SNPs} / \mathrm{kb}$ in butterflies, Külheim et al. [9] estimated 27.1 and $38.3 \mathrm{SNPs} / \mathrm{kb}$ in exons and introns, respectively, in eucalyptus, and Renaut et al. [7] found SNP densities of $>20 \mathrm{SNPs} / \mathrm{kb}$ in genes for DNA transposition, mitotic spindle organization, and biogenesis. Such findings have provided broad perspective on SNP distribution across diverse species, but all of these studies considered only the effect of sequence length variation; i.e., they estimated SNP frequencies per unit length without regard to the effect of variation in contig depth. Not unexpectedly, our data show that SNP frequency estimates are strongly and positively associated with contig length and with contig depth. Furthermore, our analyses suggest that polymorphism frequency estimates based on contig length-but-not-depth produce biased results; SNPs, $T_{i}$, and $T_{v}$ were more abundant in longer contigs assembled with more sequence reads. 
This finding suggests that both length and depth need to be considered for estimating the frequency of polymorphisms from next-generation sequence data.

A multi-model inference framework is informative for comparing the relative importance of the variables in our models. Our most robust model of frequency estimation was the global model that included contig length, depth, and transcript type. Importantly, contig depth is a more important variable than contig length when estimating SNP frequencies. This is clear from the Akaike weight $\left(w_{i}\right)$ of the models, because $w_{i}$ is best interpreted as the relative likelihood or probability of the model [31]. Consider that our global model of SNP frequency estimation was only 9.4 times more likely than the next best model (contig depth and transcript type, but no length). In stark contrast, the global model was $7.5 \times 10^{55}$ times more likely than the model of length and transcript type (i.e., no depth; Table 2). In the context of massively parallel sequencing, this suggests that SNP frequency estimation by simply correcting for contig length is inadequate. Our results support the idea that SNP frequency increases with contig length and depth, but that contig depth is a much more meaningful factor in SNP discovery.

\section{Comparison of SNP frequency between protein-coding and non-coding transcripts}

Our modeling approach can be used as a tool for inferring the intensity of selection on different categories of genes or genomic regions by comparing the SNP frequency among categories. Similar approaches have been developed for nucleotide diversity parameters such as beta $(\beta)$ [35]. We studied the distribution of SNPs with regard to the protein-coding status of an expressed transcript, but our approach should prove informative in other comparisons across genomic categories (e.g., exons versus introns, class I versus class II transposable elements, etc.).

In the current analyses, we included the dummy variable $\mathrm{C} / \mathrm{NC}$ in all candidate models to test for differences in SNP frequencies between protein-coding and non-coding transcripts. In the long dataset with 10 or more reads we found a negative coefficient of $\mathrm{C} / \mathrm{NC}$, implying SNP frequency in non-coding transcripts is higher than that in protein-coding transcripts. Our use of a poly-dT primer for cDNA synthesis may have biased our representation of the transcriptome, but a similar pattern has been reported in human genomic DNA. Zhao et al. [8] investigated the distribution of human SNPs and found that SNP frequency was higher in intronic or intergenic than in exonic regions. Our results support the hypothesis that reduced SNP frequency in protein-coding regions relative to nonprotein-coding transcripts can be attributed to the selection pressure on functionally important protein-coding regions $[15,16]$. However, absolute SNP frequency values we report here should be viewed with some caution because our transcripts were sequenced from a pool of seven tiger salamanders and pooled contig depth does not directly correspond to the actual number of haplotypes [35]. Although we cannot estimate standard nucleotide diversity parameters such as theta $(\theta)$ [36] from pooled transcripts from multiple individuals, SNP frequency by our modeling approach is still useful as a relative estimate to compare the selection pressure on different transcripts $[35,37]$.

Our data indicate there is considerable variation in SNP frequency across transcribed sequences in $A$. $t$. tigrinum. These results are consistent with the observation that different classes of functional, non-coding RNAs (e.g., microRNAs) evolve at different rates in humans and mice, with faster evolution in longer functional non-coding RNAs [18]. That said, our observed SNP frequency differences between coding and noncoding transcripts were subtle. There are several possible explanations for this. First, we relied on the CPC program [28] to parse protein-coding and non-proteincoding transcripts. CPC does so for each contig by evaluating the predicted ORF quality and the quality of the BLAST hit. This program generally performs well (accuracy of 92 96\%; [28,29], but some transcripts remain ambiguous or are falsely allocated to the wrong category. In particular, categorical assignment (coding vs. non-coding) can be limited by the number of reference genomes available for comparison [29]. The tiger salamander is not a genomic model species (e.g., no complete genome sequence is available) and as of yet has no close relatives with sequenced genomes, so this complicates the identification of orthologous sequences and protein homologs used in categorical (coding/ non-coding) transcript assignments.

A second source of bias may be the short read sequences generated by next generation sequencing. For example, some of our sequences may be derived from short sections of an untranslated region (e.g., 5' or 3' UTR) that occurs in a true protein-coding transcript. Such sequences will be erroneously categorized as noncoding transcripts due to poor ORF-related score, obfuscating SNP frequency differences between proteincoding and non-coding transcripts.

Finally, there may be true biological differences in SNP frequency between protein-coding and non-coding transcripts or within a transcript category-particularly when considering "long" transcripts. Recent analyses have revealed that even transcripts which do not encode proteins may have important functions and some of them are conserved to maintain functional domains and structures [17,38-40]. Many short non-coding transcripts such as microRNAs and small nucleolar RNAs are known to be highly conserved, functional non-coding RNAs across 
a diverse range of species [18]. In contrast, some long non-coding RNAs with a known function, such as Xist and Air, are poorly conserved across taxa $[18,41]$. This suggests rapid evolution of long non-coding RNAs despite their known functions (e.g., involved in Xchromosome dosage compensation for Xist and silencing an imprinted gene at the Igf2r locus for Air) [42,43]. Such biological differences between long and short non-coding RNAs might be reflected in our "all" contig and "long" contig datasets. We found no difference in SNP frequency between coding $v$ s. non-coding transcripts when all contigs were considered, but we did observe a trend of greater SNP density in non-coding transcripts compared to coding transcripts when only long contigs were considered. This observation provides indirect evidence that "long" non-coding transcripts have relatively fast rates of molecular evolution.

\section{Conclusions}

Our transcriptome results revealed that estimates of polymorphism frequency are affected by both length and depth of contigs, such that simply dividing the total number of SNPs by the sequence length produces biased frequency estimates. In next-generation sequencing studies where reads vary dramatically among contigs, we propose that estimates of SNP frequency should be presented as the standardized number of SNPs for a given contig length and depth instead of the current "standard" of SNPs per unit length (i.e., SNPs/ $\mathrm{kb}$ ). For example, polymorphism frequency might be reported as the number of SNPs in a $1 \mathrm{~kb}$-long transcript with coverage depth of 10 reads. Even longer and deeper contigs will become more common as sequencing technology continues to advance (so it is currently fruitless to recommend a particular read depth), but authors should at least provide readers with information about contig depth as well as length when reporting SNP frequencies.

Our modeling approach revealed that long non-coding transcripts have marginally higher SNP frequencies than protein-coding transcripts. Future models that consider more variables may further refine our ability to estimate SNP frequency, so the absolute frequency values we report herein should be viewed with some caution. Nevertheless, the relative polymorphism frequency values reported herein support the hypothesis that long noncoding transcripts are less conserved than long proteincoding transcripts, perhaps as a function of selective constraints. We think that our modeling approach (i.e., using multiple model construction and model selection approaches) can be a powerful tool for identifying selection on specific functional sequence groups by comparing the frequency and distribution of polymorphisms.

\section{Additional files}

Additional file 1: Contigs and SNPs. Information on 4,552 contigs with $\geq 10$ sequence reads and $\geq 100$ bp in length, and 2,980 SNPs found in the contigs.

Additional file 2: Supporting figures and tables. A file containing additional data: Figure S1; Figure S2; Table S1; and Table S2.

\section{Competing interests}

The authors declare no conflict of interest.

\section{Acknowledgments}

We thank Dr. Robert K. Swihart and members of the DeWoody lab for comments on this manuscript. This research was supported by a grant from the National Science Foundation (DEB-0514815) to JAD and from University Faculty Scholar funds provided through Purdue's Office of the Provost.

\section{Author details}

'Department of Forestry \& Natural Resources, Purdue University, West Lafayette, IN 47907, USA. ${ }^{2}$ Department of Biological Sciences, Purdue University, West Lafayette, IN 47907, USA. ${ }^{3}$ Current address: Department of Zoology, University of Wisconsin, Madison, WI 53706, USA.

\section{Authors' contributions}

SHE and JAD conceived and designed the experiments. SHE performed the experiments and analyzed the data. JAD contributed reagents/materials/ analysis tools. SHE and JAD wrote the manuscript. All authors read and approved the final manuscript.

Received: 9 November 2011 Accepted: 20 June 2012

Published: 20 June 2012

\section{References}

1. Chi Y-l: Homeodomain revisited: a lesson from disease-causing mutations. Hum Genet 2005, 116:433-444.

2. Ueda H, Howson JMM, Esposito L, Heward J, Snook, Chamberlain G, Rainbow DB, Hunter KMD, Smith AN, Di Genova G, et al: Association of the T-cell regulatory gene CTLA4 with susceptibility to autoimmune disease. Nature 2003, 423:506-511.

3. Nielson R: Molecular signatures of natural selection. Annu Rev Genet 2005, 39:197-218

4. Akey JM, Zhang G, Zhang K, Jin L, Shriver MD: Interrogating a high-density SNP map for signatures of natural selection. Genome Res 2002, 12:1805-1814.

5. Graur D, Li W-H: Fundamentals of molecular evolution. Sunderland, MA: Sinauer; 2000.

6. Page RDM, Holmes EC: Molecular evolution: a phylogenetic approach. Malden, MA: Blackwell; 1998.

7. Renaut S, Nolte AW, Bernatchez L: Mining transcriptomic sequences towards identifying adaptive single nucleotide polymorphisms in lake whitefish species pairs (Coregonus spp. Salmonidae). Mol Ecol 2010, 19 (Suppl. 1):115-131.

8. Zhao Z, Fu Y-X, Hewett-Emmett D, Boerwinkle E: Investigating single nucleotide polymorphism (SNP) density in the human genome and its implications for molecular evolution. Gene 2003, 312:207-213.

9. Külheim C, Hui Yeoh S, Maintz J, Foley W, Moran G: Comparative SNP diversity among four Eucalyptus species for genes from secondary metabolite biosynthetic pathways. BMC Genomics 2009, 10:452.

10. The International HapMap Consortium: A haplotype map of the human genome. Nature 2005, 437:1299-1320.

11. The International HapMap Consortium: A second generation human haplotype map of over 3.1 million SNPs. Nature 2007, 449:851-861.

12. Nordborg M, Hu T, Ishino Y, Jhaveri J, Toomajian C, Zheng H, Bakker E, Calabrese P, Gladstone J, Goyal R: The pattern of polymorphism in Arabidopsis thaliana. PLoS Biol 2005, 3:e196.

13. Barbazuk WB, Emrich SJ, Chen HD, Li L, Schnable PS: SNP discovery via 454 transcriptome sequencing. Plant J 2007, 51:910-918.

14. Vera JC, Wheat CW, Fescemyer HW, Frilander MJ, Crawford DL, Hanski I, Marden JH: Rapid transcriptome characterization for a nonmodel organism using 454 pyrosequencing. Mol Ecol 2008, 17:1636-1647. 
15. Eyre-Walker A, Keightley PD: High genome deleterious mutation rates in hominids. Nature 1999, 397:344-347.

16. Fay JC, Wyckoff GJ, Wu C-I: Positive and negative selection on the human genome. Genetics 2001, 158:1227-1234.

17. Mercer TR, Dinger ME, Mattick JS: Long non-coding RNAs: insights into functions. Nat Rev Genet 2009, 10:155-159.

18. Pang KC, Frith MC, Mattick JS: Rapid evolution of noncoding RNAs: lack of conservation does not mean lack of function. Trends Genet 2006, 22:1-5.

19. Wang J, Zhang J, Zheng H, Li J, Liu D, Li H, Samudrala R, Yu J, Wong GK-S: Mouse transcriptome: Neutral evolution of 'non-coding' complementary DNAs. Nature 2004, 431. doi:10.1038/nature03016.

20. Cheng J, Kapranov P, Drenkow J, Dike S, Brubaker S, Patel S, Long J, Stern D, Tammana $\mathrm{H}$, Helt G, et al: Transcriptional Maps of 10 Human Chromosomes at 5-Nucleotide Resolution. Science 2005, 308:1149-1154.

21. Bos DH, Gopurenko D, Williams RN, Bulut Z, DeWoody JA: Conditiondependant mate choice and a reproductive disadvantage for MHCdivergent tiger salamanders. Mol Ecol 2009, 18:3307-3315.

22. Gopurenko D, Williams RN, McCormick C, DeWoody JA: Insights into the aggregate breeding biology of the tiger salamander (Ambystoma tigrinum) as revealed by genetic parentage analyses. Mol Ecol 2006, 15:1917-1928.

23. Williams RN, DeWoody JA: Reproductive success and sexual selection in wild tiger salamanders (Ambystoma tigrinum tigrinum). Evol Biol 2009, 36:201-213.

24. Eo SH, Doyle JM, Hale MC, Marra NJ, Ruhl JD, DeWoody JA: Comparative transcriptomics and gene expression in larval tiger salamander (Ambystoma tigrinum) gill and lung tissues as revealed by pyrosequencing. Gene 2012, 492:329-338

25. Hale MC, McCormick CR, Jackson JR, DeWoody JA: Next-generation pyrosequencing of gonad transcriptomes in the polyploid lake sturgeon (Acipenser fulvescens): the relative merits of normalization and rarefaction in gene discovery. BMC Genomics 2009, 10:203.

26. Huang $X Q$, Wang JM, Aluru S, Yang SP, Hillier L: PCAP: A whole-genome assembly program. Genome Res 2003, 13:2164-2170

27. Janes DE, Chapus C, Gondo Y, Clayton DF, Sinha S, Blatti CA, Organ CL, Fujita MK, Balakrishnan CN, Edwards SV: Reptiles and mammals have differentially retained long conserved noncoding sequences from the Amniote ancestor. Genome Biol Evol 2011, 3:102-113.

28. Kong L, Zhang Y, Ye Z-Q, Liu X-Q, Zhao S-Q, Wei L, Gao G: CPC: assess the protein-coding potential of transcripts using sequence features and support vector machine. Nucleic Acids Res 2007, 35(suppl 2):W345-W349.

29. Dinger ME, Pang KC, Mercer TR, Mattick JS: Differentiating Protein-Coding and Noncoding RNA: Challenges and Ambiguities. PLoS Comput Biol 2008, 4:e1000176.

30. Akaike $\mathrm{H}$ : Information theory and an extension of the maximum likelihood principle. In Second International Symposium on Information Theory. Edited by Petrov BN, Csaki F. Budapest, Hungary: Akademiai Kiado; 1973:267-281.

31. Burnham KP, Anderson DR: Model Selection and Inference: a practical information-theoretic approach. 2nd edition. New York: Springer; 2002.

32. Torres T, Metta M, Ottenwalder B, Schlotterer C: Gene expression profiling by massively parallel sequencing. Genome Res 2007, 18:172-177.

33. Tomi P: Genome-wide allele-specific analysis: insights into regulatory variation. Nat Rev Genet 2010, 11:533-538.

34. Lipson D, Raz T, Kieu A, Jones DR, Giladi E, Thayer E, Thompson JF, Letovsky S, Milos P, Causey M: Quantification of the yeast transcriptome by singlemolecule sequencing. Nat Biotechnol 2009, 27:652-658.

35. Novaes ED, Drost R, Farmerie WG, Pappas GJ, Grattapaglia D, Sederoff RR, Kirst M: High-throughput gene and SNP discovery in Eucalyptus grandis, an uncharacterized genome. BMC Genomics 2008, 9:312.

36. Watterson GA: On the number of segregating sites in genetical models without recombination. Theor Popul Biol 1975, 7:256-276.

37. O'Neil ST, Dzurisin JDK, Carmichael RD, Lobo NF, Emrich SJ, Hellmann JJ: Population-level transcriptome sequencing of nonmodel organisms Erynnis propertius and Papilio zelicaon. BMC Genomics 2010, 11:310.

38. Ponjavic J, Ponting $\mathrm{CP}$, Lunter G: Functionality or transcriptional noise? Evidence for selection within long noncoding RNAs. Genome Res 2007 17:556-565.

39. Marques A, Ponting C: Catalogues of mammalian long noncoding RNAs: modest conservation and incompleteness. Genome Biol 2009, 10:R124.
40. Kapranov P, Cheng J, Dike S, Nix DA, Duttagupta R, Willingham AT, Stadler PF, Hertel J, Hackermüller J, Hofacker IL, et al: RNA maps reveal new RNA classes and a possible function for pervasive transcription. Science 2007, 316:14841488.

41. Nesterova TB, Slobodyanyuk SY, Elisaphenko EA, Shevchenko Al, Johnston C, Pavlova ME, Rogozin IB, Kolesnikov NN, Brockdorff N, Zakian SM: Characterization of the genomic Xist locus in rodents reveals conservation of overall gene structure and tandem repeats but rapid evolution of unique sequence. Genome Res 2001, 11:833-849.

42. Borsani G, Tonlorenzi R, Simmler MC, Dandolo L, Arnaud D, Capra V, Grompe M, Pizzuti A, Muzny D, Lawrence C, et al: Characterization of a murine gene expressed from the inactive $\mathbf{X}$ chromosome. Nature 1991, 351:325-329.

43. Sleutels F, Zwart R, Barlow DP: The non-coding Air RNA is required for silencing autosomal imprinted genes. Nature 2002, 415:810-813.

doi:10.1186/1471-2164-13-259

Cite this article as: Eo and DeWoody: The effects of contig length and depth on the estimation of SNP frequencies, and the relative abundance of SNPs in protein-coding and non-coding transcripts of tiger salamanders (Ambystoma tigrinum). BMC Genomics 2012 13:259.

\section{Submit your next manuscript to BioMed Central and take full advantage of:}

- Convenient online submission

- Thorough peer review

- No space constraints or color figure charges

- Immediate publication on acceptance

- Inclusion in PubMed, CAS, Scopus and Google Scholar

- Research which is freely available for redistribution 